\title{
The impact of obesity on perioperative complications in patients undergoing anterior lumbar interbody fusion
}

\author{
${ }^{*}$ Michael M. Safaee, MD, ${ }^{1}$ Alexander Tenorio, BA, ${ }^{1}$ Joseph A. Osorio, MD, PhD, ${ }^{1}$ \\ Winward Choy, MD,, Dominic Amara, BA, ${ }^{1}$ Lillian Lai, BS, ${ }^{1}$ Annette M. Molinaro, PhD, ${ }^{1}$ \\ Yalan Zhang, MS, ${ }^{1}$ Serena S. Hu, MD, ${ }^{2}$ Bobby Tay, MD, ${ }^{3}$ Shane Burch, MD, ${ }^{3}$ Sigurd H. Berven, MD, ${ }^{3}$ \\ Vedat Deviren, MD, ${ }^{3}$ Sanjay S. Dhall, MD, ${ }^{1}$ Dean Chou, MD, ${ }^{1}$ Praveen V. Mummaneni, MD, ${ }^{1}$ \\ Charles M. Eichler, MD, ${ }^{4}$ Christopher P. Ames, MD, ${ }^{1}$ and Aaron J. Clark, MD, PhD ${ }^{1}$
}

1Department of Neurological Surgery, University of California, San Francisco; ${ }^{2}$ Department of Orthopedic Surgery, Stanford University, Palo Alto; and Departments of ${ }^{3}$ Orthopedic Surgery and ${ }^{4}$ Vascular Surgery, University of California, San Francisco, California

\begin{abstract}
OBJECTIVE Anterior approaches to the lumbar spine provide wide exposure that facilitates placement of large grafts with high fusion rates. There are limited data on the effects of obesity on perioperative complications.

METHODS Data from consecutive patients undergoing anterior lumbar interbody fusion (ALIF) from 2007 to 2016 at a single academic center were analyzed. The primary outcome was any perioperative complication. Complications were divided into those occurring intraoperatively and those occurring postoperatively. Multivariate logistic regression was used to assess the association of obesity and other variables with these complications. An estimation table was used to identify a body mass index (BMI) threshold associated with increased risk of postoperative complication.

RESULTS A total of 938 patients were identified, and the mean age was 57 years; 511 were females (54.5\%). The mean BMI was $28.7 \mathrm{~kg} / \mathrm{m}^{2}$, with $354(37.7 \%)$ patients classified as obese $\left(\mathrm{BMI} \geq 30 \mathrm{~kg} / \mathrm{m}^{2}\right)$. Forty patients $(4.3 \%)$ underwent a lateral transthoracic approach, while the remaining 898 (95.7\%) underwent a transabdominal retroperitoneal approach. Among patients undergoing transabdominal retroperitoneal ALIF, complication rates were higher for obese patients than for nonobese patients $(37.0 \%$ vs $28.7 \%, p=0.010)$, a difference that was driven primarily by postoperative complications $(36.1 \%$ vs $26.0 \%, p=0.001)$ rather than intraoperative complications $(3.2 \%$ vs $4.3 \%, p=0.416)$. Obese patients had higher rates of ileus $(11.7 \%$ vs $7.2 \%, p=0.020)$, wound complications $(11.4 \%$ vs $3.4 \%, p<0.001)$, and urinary tract infections (UTI) (5.0\% vs $2.5 \%, p=0.049)$. In a multivariate model, age, obesity, and number of ALIF levels fused were associated with an increased risk of postoperative complication. An estimation table including 19 candidate cut-points, odds ratios, and adjusted $p$ values found a BMI $\geq 31 \mathrm{~kg} / \mathrm{m}^{2}$ to have the highest association with postoperative complication $(p=0.012)$.
\end{abstract}

CONCLUSIONS Obesity is associated with increased postoperative complications in ALIF, including ileus, wound complications, and UTI. ALIF is a safe and effective procedure. However, patients with a BMI $\geq 31 \mathrm{~kg} / \mathrm{m}^{2}$ should be counseled on their increased risks and warrant careful preoperative medical optimization and close monitoring in the postoperative setting.

https://thejns.org/doi/abs/10.3171/2020.2.SPINE191418

KEYWORDS lumbar fusion; anterior approach; anterior lumbar interbody fusion; body mass index; obesity; perioperative complications

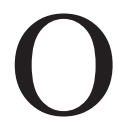
BESITY is a significant public health concern associated with increased risk of several long-term health problems, including disc degeneration and lowback pain. ${ }^{1}$ In cases of severe degeneration, instability, or deformity, patients may require interbody fusion. A variety of options exist, including posterior lumbar interbody fusion, transforaminal lumbar interbody fusion, direct lateral lumbar interbody fusion, oblique lumbar interbody fusion, and anterior lumbar interbody fusion (ALIF). ${ }^{2}$ Obesity can increase the technical difficulty of these operations due to

ABBREVIATIONS ALIF = anterior lumbar interbody fusion; $\mathrm{BMI}=$ body mass index; $\mathrm{BMP}=$ bone morphogenetic protein; $\mathrm{CCl}=\mathrm{Charlson} \mathrm{Comorbidity} \mathrm{Index;} \mathrm{DVT}=$ deep venous thrombosis; $\mathrm{EBL}=$ estimated blood loss; $\mathrm{LOS}=$ length of stay; $\mathrm{PE}=$ pulmonary embolism; UTI = urinary tract infection.

SUBMITTED November 25, 2019. ACCEPTED February 27, 2020.

INCLUDE WHEN CITING Published online April 24, 2020; DOI: 10.3171/2020.2.SPINE191418.

${ }^{*}$ M.M.S. and A.T. contributed equally to this work. 
challenges associated with exposure, operative time, and blood loss. ${ }^{3-7}$ While the literature has established an association between increased complications and obesity in patients undergoing posterior spinal fusions, ${ }^{1,3,8-11}$ few have reported on surgical complications after ALIF. ${ }^{12-14}$

The ALIF procedure has become an increasingly popular technique because it provides wide access and visualization of the disc space, which facilitates a complete discectomy, and it can accommodate a large, lordotic interbody graft that is associated with high fusion rates. ${ }^{15}$ Additionally, several studies have reported improved restoration of lumbar lordosis and disc height compared to other interbody techniques. ${ }^{16-18}$ Disadvantages include the need for an access surgeon, increased risk of vascular injury, inability to easily access higher vertebral levels, and the frequent need for a secondary procedure for posterior stabilization. ${ }^{17}$ There are limited data on the effect of obesity and ALIF-related perioperative complications, with no studies focusing specifically on immediate postoperative complications. ${ }^{12}$

Given the increased popularity and utilization of ALIF, understanding the role obesity plays in the perioperative risk profile for this surgery is critical. To evaluate this effect, we performed a retrospective review of experience at a single tertiary care center, with a specific goal of determining potential associations between body mass index (BMI), obesity, and perioperative complications among patients undergoing ALIF. Furthermore, we distinguished intraoperative from immediate postoperative complications to control for the unique risks associated with the ALIF exposure.

\section{Methods}

\section{Patient Identification}

We performed a retrospective review of consecutive patients who underwent ALIF from 2007 to 2016 at the University of California, San Francisco Medical Center. All activities were approved by our institutional review board. Exclusion criteria included patients under 18 years of age, patients with insufficient data to calculate BMI, and patients undergoing revision ALIF. Additionally, patients undergoing lateral transpsoas approaches were excluded. All transabdominal retroperitoneal or lateral transthoracic exposures were performed by a fellowship-trained vascular surgeon.

\section{Data Collection}

Patient demographics and surgical characteristics were collected through a review of the institutional electronic medical records system. Variables collected included age, sex, BMI, obesity (defined using the World Health Organization classification of $\mathrm{BMI} \geq 30$ ), Charlson Comorbidity Index (CCI), smoking status, and presence of diabetes. Surgical characteristics were collected, including surgical approach (transabdominal retroperitoneal or lateral transthoracic), number of levels fused, anterior uppermost level, procedure duration, estimated blood loss (EBL), presence of a second-stage surgery during the same admission, length of stay (LOS), and follow-up duration.

Surgical complications were categorized as intraopera- tive or postoperative. Intraoperative complications were classified as arterial, venous, ureteral, bowel, or medical. Postoperative complications were classified as ileus, wound-related (dehiscence, hernia, or infection), neurological (new postoperative neurological symptom or deficit), ALIF-related hematoma, or medical. Postoperative medical complications were further subclassified as pulmonary (pneumonia, pleural effusion, pneumothorax, pulmonary edema, exacerbation of chronic obstructive pulmonary disease, hypoxia, or respiratory failure requiring intubation), cardiac (arrhythmia, congestive heart failure, myocardial infarction, or orthostatic hypotension), urological (urinary retention, incontinence, epididymitis, scrotal edema, or retrograde ejaculation), renal (acute kidney injury or acute renal failure), urinary tract infection (UTI), and thromboembolic events, including deep venous thrombosis (DVT) or pulmonary embolism (PE).

\section{Statistical Analysis}

Univariate analysis was performed using the chisquare test for categorical variables and the Student t-test for continuous variables. A multivariate model was generated using binary logistic regression. Variables with a $\mathrm{p}$ value $<0.10$ on univariate analysis were included in the multivariate model with backward elimination. Statistical significance was defined as $\mathrm{p}<0.050$. To assess the associations of BMI with the risk of perioperative complications, several approaches were applied to find the optimal cut-points for BMI, including smoothing splines, grouped data plots, and an estimation table of optimal cut-points. The grouped data plot was created by grouping BMI into deciles and plotting the mean value of BMI within each decile against the proportion of patients with perioperative complications in that decile.

\section{Results}

\section{Patient Demographics}

A total of 938 patients met inclusion criteria (898 who underwent a transabdominal retroperitoneal approach and 40 who underwent a lateral transthoracic approach). The patients' mean age was 57 years (range 18-85 years), and $511(54.5 \%)$ of the patients were women. The mean CCI score was 0.8 , and the mean BMI was $28.7 \mathrm{~kg} / \mathrm{m}^{2}$ (range $16.4-49.7 \mathrm{~kg} / \mathrm{m}^{2}$ ). Using BMI $\geq 30 \mathrm{~kg} / \mathrm{m}^{2}$, we identified 354 obese patients $(37.7 \%)$. There were 117 smokers (12.5\%), 99 diabetics (10.6\%), and 540 patients (57.6\%) with a history of spine surgery. The mean number of levels fused by ALIF was 1.9 (range 1-8), with a distribution of upper levels as follows: $8(0.9 \%)$ at T10-12, $15(1.6 \%)$ at $\mathrm{L} 1,35(3.7 \%)$ at $\mathrm{L} 2,195(20.8 \%)$ at $\mathrm{L} 3,412(43.9 \%)$ at $\mathrm{L} 4$, and $273(29.1 \%)$ at L5, including one case of S1-2 ALIF. A lateral transthoracic approach was used in 40 cases (4.3\%), and a transabdominal retroperitoneal approach was used in the remaining 898 cases (95.6\%). The mean operative duration was 253 minutes, and the mean EBL was $364 \mathrm{ml}$. Two-stage surgeries (anterior-posterior fusions performed on separate days) were performed in 778 cases (82.9\%). The mean LOS was 7 days, and the mean follow-up duration was 27 months. Patient demographics are summarized in Table 1. 
TABLE 1. Demographics and surgical characteristics in 938 patients

\begin{tabular}{|c|c|}
\hline Characteristic & Value \\
\hline \multicolumn{2}{|l|}{ Age, yrs } \\
\hline Mean (range) & $57(18-85)$ \\
\hline Median (IQR) & $59(50-67)$ \\
\hline No. of females & $511(54.5 \%)$ \\
\hline \multicolumn{2}{|l|}{$\mathrm{BMI}, \mathrm{kg} / \mathrm{m}^{2}$} \\
\hline Mean (range) & $28.7(16.4-49.7)$ \\
\hline Median (IQR) & $28.1(24.3-32.3)$ \\
\hline Obese $(\mathrm{BMI} \geq 30)$ & $354(37.7 \%)$ \\
\hline \multicolumn{2}{|l|}{$\mathrm{CCl}$ score } \\
\hline Mean (range) & $0.8(0-11)$ \\
\hline Median (IQR) & $0(0-1)$ \\
\hline Diabetic & $99(10.6 \%)$ \\
\hline Current smoker & $117(12.5 \%)$ \\
\hline Previous spine surgery & $540(57.6 \%)$ \\
\hline \multicolumn{2}{|l|}{ Indication for surgery } \\
\hline Degenerative/spondylolisthesis & $470(50.1 \%)$ \\
\hline Deformity & $317(33.8 \%)$ \\
\hline Infection & $43(4.6 \%)$ \\
\hline Trauma & $7(0.7 \%)$ \\
\hline Tumor & $13(1.4 \%)$ \\
\hline Pseudarthrosis & $88(9.4 \%)$ \\
\hline \multicolumn{2}{|l|}{ No. of anterior levels fused } \\
\hline 1 & $350(37.3 \%)$ \\
\hline 2 & $396(42.2 \%)$ \\
\hline 3 & $164(17.5 \%)$ \\
\hline$\geq 4$ & $28(3.0 \%)$ \\
\hline Lateral transthoracic approach & $40(4.3 \%)$ \\
\hline \multicolumn{2}{|l|}{ Anterior upper level } \\
\hline T10-12 & $8(0.9 \%)$ \\
\hline L1 & $15(1.6 \%)$ \\
\hline L2 & $35(3.7 \%)$ \\
\hline L3 & $195(20.8 \%)$ \\
\hline L4 & $412(43.9 \%)$ \\
\hline $\mathrm{L}^{*}$ & $273(29.1 \%)$ \\
\hline \multicolumn{2}{|l|}{ Operative duration, mins } \\
\hline Mean (range) & $253(58-823)$ \\
\hline Median (IQR) & $211(137-341)$ \\
\hline \multicolumn{2}{|l|}{$\mathrm{EBL}, \mathrm{ml}$} \\
\hline Mean (range) & $364(20-12,000)$ \\
\hline Median (IQR) & $200(100-300)$ \\
\hline Two-stage surgery & $778(82.9 \%)$ \\
\hline Any surgical complication & $311(33.2 \%)$ \\
\hline Intraoperative & $40(4.3 \%)$ \\
\hline Postoperative & $291(31.0 \%)$ \\
\hline \multicolumn{2}{|l|}{ LOS, days } \\
\hline Mean (range) & $7(1-71)$ \\
\hline Median (IQR) & $6(4-9)$ \\
\hline \multicolumn{2}{|l|}{ Follow-up duration, mos } \\
\hline Mean (range) & $27(0-155)$ \\
\hline Median (IQR) & $16(8-9)$ \\
\hline
\end{tabular}

\section{Surgical Complications}

Surgical complications were identified in 311 patients (33.2\%). This included 40 patients with intraoperative complications (4.3\%) and 291 patients with postoperative complications $(31.0 \%)$. We compared patients with any perioperative complication to those without a perioperative complication. Patients with a complication were older (61 vs 56 years, $\mathrm{p}<0.001$ ) and had higher CCI score distributions $(p=0.004)$. There were no differences in sex or smoking status. Among patients with surgical complications, there was a higher rate of obesity $(43.1 \%$ vs $35.1 \%$, $\mathrm{p}=0.017)$. Patients with any complication had a higher mean number of levels fused $(2.1$ vs $1.8, \mathrm{p}<0.001)$ and a higher rate of bone morphogenetic protein (BMP) use $(57.6 \%$ vs $48.2 \%, p=0.007)$. There was a higher proportion of patients who underwent a lateral transthoracic approach in the surgical complication group $(8.0 \%$ vs $2.4 \%$, $\mathrm{p}<0.001)$. Operative duration was slightly longer for patients with a complication (262 vs 248 minutes, $\mathrm{p}=0.170$ ), with significantly higher EBL (556 vs $260 \mathrm{ml}, \mathrm{p}<0.001$ ). LOS was also longer for patients with a complication (10.4 vs 6.1 days, $\mathrm{p}<0.001)$. There were no significant differences in rates of two-stage surgery or length of follow-up. Additional data are summarized in Table 2.

\section{Comparison of Complications by Approach}

We compared perioperative complications between transabdominal retroperitoneal and lateral transthoracic approaches. Patients with an indication of an infection (30.0\% vs $3.5 \%, \mathrm{p}<0.001)$, trauma $(10.0 \%$ vs $0.3 \%$, p < $0.01)$, and tumor $(17.5 \%$ vs $0.7 \%, p<0.001)$ were more likely to undergo a lateral transthoracic approach, while transabdominal retroperitoneal approaches were more common among patients with degenerative disease $(51.8 \%$ vs $12.5 \%, \mathrm{p}<0.001)$ or deformity $(34.3 \%$ vs $22.5 \%$, p = $0.123)$. Complications were more common among patients in whom lateral transthoracic approaches were used $(62.5 \%$ vs $31.8 \%, \mathrm{p}<0.001)$, most notably higher rates of wound complications $(17.5 \%$ vs $6.5 \%, \mathrm{p}=0.017)$, hematoma $(7.5 \%$ vs $1.1 \%, \mathrm{p}=0.015)$, and pulmonary complications $(20.0 \%$ vs $5.7 \%, \mathrm{p}=0.002)$. These results are summarized in Table 3.

\section{Complications Among Obese Patients}

We compared demographic and surgical characteristics among obese and nonobese patients who underwent transabdominal retroperitoneal ALIF. There were no significant differences in age, sex, smoking status, or history of spine surgery; however, there was a higher proportion of diabetic patients in the obese group $(17.9 \%$ vs $5.7 \%$, p $<0.001)$. The mean number of anterior levels fused and anterior upper level were not significantly different. There were no differences in BMP use, operative duration, EBL, use of two-stage surgery, LOS, or follow-up duration. These results are summarized in Table 4.

Given the well-matched surgical characteristics between obese and nonobese patients undergoing transabdominal retroperitoneal ALIF, we subsequently performed a comparison of complications between these groups. The cumulative intraoperative complication rate 
TABLE 2. Comparison of patients with and without surgical complications

\begin{tabular}{|c|c|c|c|}
\hline Variable & No Surgical Complication $(n=627)$ & Any Surgical Complication $(n=311)$ & p Value \\
\hline Age, yrs & 56 & 61 & $<0.001$ \\
\hline Female sex & $338(53.9 \%)$ & $173(55.6 \%)$ & 0.619 \\
\hline $\mathrm{BMI}, \mathrm{kg} / \mathrm{m}^{2}$ & 28.3 & 29.3 & 0.013 \\
\hline Obese $\left(\mathrm{BMI} \geq 30 \mathrm{~kg} / \mathrm{m}^{2}\right)$ & $220(35.1 \%)$ & $134(43.1 \%)$ & 0.017 \\
\hline \multicolumn{4}{|l|}{$\mathrm{CCl}$ score } \\
\hline $0-2$ & $585(93.3 \%)$ & $273(87.8 \%)$ & 0.004 \\
\hline $3-5$ & $29(4.6 \%)$ & $25(8.0 \%)$ & 0.035 \\
\hline$\geq 6$ & $13(2.1 \%)$ & $13(4.2 \%)$ & 0.064 \\
\hline Diabetic & $63(10 \%)$ & $36(11.6 \%)$ & 0.473 \\
\hline Current smoker & $71(11.3 \%)$ & $46(14.8 \%)$ & 0.130 \\
\hline Previous spine surgery & $353(56.4 \%)$ & $187(60.1 \%)$ & 0.264 \\
\hline \multicolumn{4}{|l|}{ Surgical indication } \\
\hline Degenerative/spondylolisthesis & $352(56.1 \%)$ & $118(37.9 \%)$ & $<0.001$ \\
\hline Deformity & $185(29.5 \%)$ & $132(42.4 \%)$ & $<0.001$ \\
\hline Infection & $21(3.3 \%)$ & $22(7.1 \%)$ & 0.010 \\
\hline Trauma & $3(0.5 \%)$ & $4(1.3 \%)$ & 0.176 \\
\hline Tumor & $7(1.1 \%)$ & $6(1.9 \%)$ & 0.682 \\
\hline Pseudarthrosis & $59(9.4 \%)$ & $29(9.3 \%)$ & 0.966 \\
\hline Mean no. of anterior levels fused & 1.8 & 2.1 & $<0.001$ \\
\hline Transthoracic approach & $15(2.4 \%)$ & $25(8.0 \%)$ & $<0.001$ \\
\hline \multicolumn{4}{|l|}{ Anterior upper level } \\
\hline T10-12 & $4(0.6 \%)$ & $4(1.3 \%)$ & $0.451^{*}$ \\
\hline L1 & $3(0.5 \%)$ & $11(3.5 \%)$ & $0.001^{*}$ \\
\hline L2 & $17(2.7 \%)$ & $18(5.8 \%)$ & 0.019 \\
\hline L3 & $110(17.5 \%)$ & $86(27.7 \%)$ & $<0.001$ \\
\hline L4 & $280(44.7 \%)$ & $132(42.4 \%)$ & 0.520 \\
\hline L5 & $213(34.0 \%)$ & $60(19.3 \%)$ & $<0.001$ \\
\hline Use of BMP & $302(48.2 \%)$ & $179(57.6 \%)$ & 0.007 \\
\hline Mean operative duration, mins & 248 & 262 & 0.170 \\
\hline Mean EBL, ml & 260 & 556 & $<0.001$ \\
\hline Two-stage surgery & $511(81.5 \%)$ & $267(85.9 \%)$ & 0.095 \\
\hline Mean LOS, days & 6.1 & 10.4 & $<0.001$ \\
\hline Mean follow-up, mos & 25.9 & 28.1 & 0.221 \\
\hline
\end{tabular}

was $3.9 \%$, with no significant difference between obese and nonobese patients $(3.2 \%$ vs $4.3 \%, p=0.416)$. This included no difference in rates of intraoperative arterial, venous, ureteral, bowel, or medical complications. There were no cases of intraoperative bowel or ureteral injury. Among postoperative complications, the rate for the entire cohort was $29.8 \%$, with a significantly higher rate among obese versus nonobese patients (36.1\% vs $26.0 \%$, $\mathrm{p}=0.001)$. The most significant differences involved increased rates of ileus $(11.7 \%$ vs $7.2 \%, \mathrm{p}=0.020)$, anterior wound-related complications $(11.4 \%$ vs $3.4 \%, \mathrm{p}<0.001)$, and UTI $(5.0 \%$ vs $2.5 \%, \mathrm{p}=0.049)$. Among wound-related complications, significant differences were found in rates of dehiscence (3.8\% vs $0.5 \%, \mathrm{p}<0.001)$, hernia (5.3\% vs $2.0 \%, \mathrm{p}=0.007)$, and infection (3.2\% vs $1.1 \%, \mathrm{p}=0.022)$.
There were no significant differences in postoperative neurological deficits, hematomas, DVT/PE, or other medical complications, including pulmonary, cardiac, urologi$\mathrm{cal}$, and renal complications. We also performed a comparison of complications across obesity subgroups based on BMI classification, including obese (BMI $30-34.9 \mathrm{~kg} /$ $\mathrm{m}^{2}$ ), severely obese (BMI $35-39.9 \mathrm{~kg} / \mathrm{m}^{2}$ ), and morbidly obese $\left(\mathrm{BMI}>40 \mathrm{~kg} / \mathrm{m}^{2}\right)$. This included 203 obese, $102 \mathrm{se}-$ verely obese, and 36 morbidly obese patients. Intraoperative complications were similar across groups. Postoperative complications were also similar across these groups. There was a trend toward increased rate of hematomas ( $\mathrm{p}$ $=0.052)$ and DVT/PE $(p=0.054)$ in morbidly obese compared to obese and severely obese patients. These results are summarized in Table 5. 
TABLE 3. Comparison of perioperative complications by ALIF approach

\begin{tabular}{|c|c|c|c|}
\hline Variable & Anterior Retroperitoneal $(n=898)$ & Lateral Transthoracic $(n=40)$ & $p$ Value \\
\hline \multicolumn{4}{|l|}{ Surgical indication } \\
\hline Degenerative/spondylolisthesis & $465(51.8 \%)$ & $5(12.5 \%)$ & $<0.001$ \\
\hline Deformity & $308(34.3 \%)$ & $9(22.5 \%)$ & 0.123 \\
\hline Infection & $31(3.5 \%)$ & $12(30.0 \%)$ & $<0.001^{*}$ \\
\hline Trauma & $3(0.3 \%)$ & $4(10.0 \%)$ & $<0.001^{*}$ \\
\hline Tumor & $6(0.7 \%)$ & $7(17.5 \%)$ & $<0.001^{*}$ \\
\hline Pseudarthrosis & $85(9.5 \%)$ & $3(7.5 \%)$ & $1.000^{*}$ \\
\hline Any complication & $286(31.8 \%)$ & $25(62.5 \%)$ & $<0.001$ \\
\hline Intraoperative complication & $35(3.9 \%)$ & $5(12.5 \%)$ & $0.024^{*}$ \\
\hline Arterial & $2(0.2 \%)$ & $0(0 \%)$ & $1.000^{*}$ \\
\hline Venous & $32(3.6 \%)$ & $3(7.5 \%)$ & $0.184^{*}$ \\
\hline Ureteral & $0(0 \%)$ & $1(2.5 \%)$ & $0.043^{*}$ \\
\hline Bowel & $0(0 \%)$ & $0(0 \%)$ & NA \\
\hline Medical & $1(0.1 \%)$ & $1(2.5 \%)$ & $0.084^{*}$ \\
\hline Postoperative complication & $268(29.8 \%)$ & $23(57.5 \%)$ & $<0.001$ \\
\hline lleus & $80(8.9 \%)$ & $4(10.0 \%)$ & $0.776^{*}$ \\
\hline Wound & $58(6.5 \%)$ & $7(17.5 \%)$ & $0.017^{*}$ \\
\hline Dehiscence & $16(1.8 \%)$ & $0(0 \%)$ & $1.000^{*}$ \\
\hline Hernia & $29(3.2 \%)$ & $3(7.5 \%)$ & $0.152^{*}$ \\
\hline Infection & $17(1.9 \%)$ & $4(10.0 \%)$ & $0.010^{*}$ \\
\hline Neurological & $3(0.3 \%)$ & $0(0 \%)$ & $1.000^{*}$ \\
\hline Hematoma & $10(1.1 \%)$ & $3(7.5 \%)$ & $0.015^{*}$ \\
\hline Medical & $168(18.7 \%)$ & $13(32.5 \%)$ & 0.031 \\
\hline Pulmonary & $51(5.7 \%)$ & $8(20.0 \%)$ & 0.002 \\
\hline Cardiac & $41(4.6 \%)$ & $4(10.0 \%)$ & $0.120^{*}$ \\
\hline Renal & $16(1.8 \%)$ & $0(0 \%)$ & $1.000^{*}$ \\
\hline Urologic & $42(4.7 \%)$ & $0(0 \%)$ & $0.252^{*}$ \\
\hline UTI & $31(3.5 \%)$ & $1(2.5 \%)$ & $1.000^{*}$ \\
\hline DVT/PE & $19(2.1 \%)$ & $2(5.0 \%)$ & $0.224^{*}$ \\
\hline
\end{tabular}

\section{Multivariate Analysis for Postoperative Complications}

We performed multivariate binary logistic regression to determine the association of specific variables with postoperative complications using variables that demonstrated a significance $(\mathrm{p}<0.100)$ by univariate analysis. Lateral transthoracic approaches were excluded. The model included age, obesity, CCI score of 3 or more, use of BMP, number of ALIF levels fused, EBL, use of a second-stage surgery, anterior upper level at L3 or above, and an indication of degenerative disease/spondylolisthesis, deformity, or osteomyelitis. Variables with a significant association with postoperative complications included age (OR 1.026, 95\% CI $1.010-1.043, \mathrm{p}=0.001)$, obesity (OR $1.672,95 \%$ CI $1.177-2.376, p=0.004)$, number of levels fused (OR $1.319,95 \%$ CI $1.046-1.663, \mathrm{p}=0.019)$, and surgical indication of degenerative disease/spondylolisthesis (OR 0.625, 95\% CI 0.430-0.907, $\mathrm{p}=0.004)$. These results are summarized in Table 6.

\section{BMI Threshold for Postoperative Complications}

In the univariate smoothing spline with BMI and odds ratio of postoperative complication, as well as the multivariate model including age, BMI, number of fused levels, CCI score, use of BMP, and operative duration, there was no identifiable BMI threshold associated with increase postoperative complications. No appropriate threshold was identified in the grouped data plot either. A subsequent estimation table was generated to include 19 candidate cut-points, odds ratios, and corresponding unadjusted and adjusted $\mathrm{p}$ values using Bonferroni correction. A BMI threshold of 31 had the lowest unadjusted $p$ value $(p<$ $0.001)$ and adjusted $\mathrm{p}$ value $(\mathrm{p}=0.012)$ shown in the forest plot (Fig. 1).

\section{Discussion}

ALIF is an increasingly utilized technique for a variety of lumbar pathologies. As its use increases, it is important 
TABLE 4. Comparison of obese and nonobese patients who underwent transabdominal retroperitoneal ALIF

\begin{tabular}{|c|c|c|c|}
\hline Variable & Nonobese Patients $(n=557)$ & Obese Patients $(n=341)$ & $p$ Value \\
\hline Age, yrs & 58 & 57 & 0.094 \\
\hline Female sex & $319(57.3 \%)$ & $180(52.8 \%)$ & 0.189 \\
\hline Mean BMI, kg/m² & 25.0 & 34.7 & $<0.001$ \\
\hline \multicolumn{4}{|l|}{ CCl score } \\
\hline $0-2$ & $520(93.4 \%)$ & $314(92.1 \%)$ & 0.471 \\
\hline $3-5$ & $20(3.6 \%)$ & $24(7.0 \%)$ & 0.020 \\
\hline$\geq 6$ & $17(3.1 \%)$ & $3(0.9 \%)$ & 0.032 \\
\hline Diabetic & $32(5.7 \%)$ & $61(17.9 \%)$ & $<0.001$ \\
\hline Current smoker & $77(13.8 \%)$ & $36(10.6 \%)$ & 0.152 \\
\hline Previous spine surgery & $310(55.7 \%)$ & $207(60.7 \%)$ & 0.137 \\
\hline \multicolumn{4}{|l|}{ Surgical indication } \\
\hline Degenerative/spondylolisthesis & $280(50.3 \%)$ & $185(54.3 \%)$ & 0.246 \\
\hline Deformity & $205(36.8 \%)$ & $103(30.2 \%)$ & 0.043 \\
\hline Infection & $19(3.4 \%)$ & $12(3.5 \%)$ & 0.931 \\
\hline Trauma & $3(0.5 \%)$ & $0(0 \%)$ & 0.175 \\
\hline Tumor & $4(0.7 \%)$ & $2(0.6 \%)$ & 0.814 \\
\hline Pseudarthrosis & $46(8.3 \%)$ & $39(11.4 \%)$ & 0.114 \\
\hline Mean no. of anterior levels fused & 1.8 & 1.8 & 0.475 \\
\hline \multicolumn{4}{|l|}{ Anterior upper level } \\
\hline $\mathrm{L} 1$ & $1(0.2 \%)$ & $0(0 \%)$ & $1.000^{*}$ \\
\hline L2 & $17(3.1 \%)$ & $6(1.8 \%)$ & 0.234 \\
\hline L3 & $113(20.3 \%)$ & $76(22.3 \%)$ & 0.475 \\
\hline L4 & $255(45.8 \%)$ & $157(46.0 \%)$ & 0.939 \\
\hline L5 & $171(30.7 \%)$ & $102(29.9 \%)$ & 0.803 \\
\hline Use of BMP & $294(52.8 \%)$ & $174(51.0 \%)$ & 0.609 \\
\hline Mean operative duration, mins & 246 & 256 & 0.295 \\
\hline Mean EBL, ml & 321 & 348 & 0.665 \\
\hline Two-stage surgery & $462(82.9 \%)$ & $283(83.0 \%)$ & 0.986 \\
\hline Mean LOS, days & 7.1 & 7.4 & 0.473 \\
\hline Mean follow-up, mos & 27.0 & 25.5 & 0.394 \\
\hline
\end{tabular}

Unless otherwise specified, values are presented as the number (\%) of patients.

${ }^{*}$ Fisher's exact test.

to characterize the factors that may increase the risk of surgical complications. Obesity has been a well-studied determinant of surgical complications in spinal surgery. ${ }^{3}$ Few have looked at the anterior approach exclusively and have been limited by their small sample sizes. ${ }^{12-14}$ Peng et al. examined 74 patients undergoing anterior lumbar surgery and found comparable rates of perioperative complications between obese and nonobese patients..$^{13}$ Additionally, Lucas et al. reviewed 84 patients undergoing ALIF and found no increase in infectious or vascular complications among overweight and obese patients compared to normal-weight individuals. In our study of 898 patients who underwent transabdominal retroperitoneal ALIF, we found an increase in perioperative surgical complications in obese patients compared to nonobese patients $(37.0 \%$ vs $28.7 \%, \mathrm{p}=0.010$ ), a difference that was driven primarily by postoperative complications $(36.1 \%$ vs $26.0 \%$, $p=$ $0.001)$, rather than intraoperative complications $(3.2 \%$ vs $4.3 \%, \mathrm{p}=0.416)$. These findings remained consistent in our multivariate analysis, as obesity demonstrated a significant association with postoperative complications (OR $1.672,95 \%$ CI 1.177-2.376, $p=0.004)$. Since the goals of this study were to investigate transabdominal retroperitoneal ALIF, we excluded lateral transpsoas, prepsoas, and transthoracic approaches in our formal analysis given the significantly different surgical indications and risk profiles associated with these approaches.

The most common complication was ileus (8.9\%), with a higher rate among obese patients (11.7\% vs $7.2 \%)$. Durand et al. analyzed a cohort of 59,410 patients undergoing deformity correction surgery and found a similar increased risk of ileus with anterior surgical approaches. ${ }^{19}$ Patients are often on high doses of opioids in the perioperative period, thus exacerbating the risk of ileus. The risk is also likely higher in obese patients given the more extensive mobilization and forceful retraction of peritoneal contents in order to provide adequate exposure. Ileus can significantly prolong hospital stays, and although we were 
TABLE 5. Summary of surgical complications among patients who underwent transabdominal retroperitoneal ALIF

\begin{tabular}{|c|c|c|c|c|c|c|c|c|}
\hline Variable & $\begin{array}{l}\text { All Patients } \\
(\mathrm{n}=898)\end{array}$ & $\begin{array}{c}\text { Nonobese } \\
\text { Patients w/ BMI } \\
<30 \mathrm{~kg} / \mathrm{m}^{2} \\
(\mathrm{n}=557)\end{array}$ & $\begin{array}{c}\text { All Obese } \\
\text { Patients w/ BMl } \\
>30 \mathrm{~kg} / \mathrm{m}^{2} \\
(\mathrm{n}=341)\end{array}$ & $\begin{array}{c}p \\
\text { Value }\end{array}$ & $\begin{array}{l}\text { Obese Patients } \\
\text { w/ BMl } \\
30-34.9 \mathrm{~kg} / \mathrm{m}^{2} \\
(\mathrm{n}=203)\end{array}$ & $\begin{array}{c}\text { Severely Obese } \\
\text { Patients w/ BMl } \\
35-39.9 \mathrm{~kg} / \mathrm{m}^{2} \\
(n=102)\end{array}$ & $\begin{array}{l}\text { Morbidly Obese } \\
\text { Patients w/ BMl } \\
>40 \mathrm{~kg} / \mathrm{m}^{2} \\
(\mathrm{n}=36)\end{array}$ & $\begin{array}{c}p \\
\text { Value }\end{array}$ \\
\hline Any complication & $286(31.8 \%)$ & $160(28.7 \%)$ & $126(37.0 \%)$ & 0.010 & $74(36.5 \%)$ & $39(38.2 \%)$ & $13(36.1 \%)$ & 0.949 \\
\hline Intraop complication & $35(3.9 \%)$ & $24(4.3 \%)$ & $11(3.2 \%)$ & 0.416 & $6(3.0 \%)$ & $2(2.0 \%)$ & $3(8.3 \%)$ & $0.136^{*}$ \\
\hline Arterial & $2(0.2 \%)$ & $2(0.4 \%)$ & $0(0 \%)$ & $0.528^{*}$ & $0(0 \%)$ & $0(0 \%)$ & $0(0 \%)$ & NA \\
\hline Venous & $32(3.6 \%)$ & $21(3.8 \%)$ & $11(3.2 \%)$ & 0.669 & $6(3.0 \%)$ & $2(2.0 \%)$ & $3(8.3 \%)$ & $0.136^{*}$ \\
\hline Ureteral & $0(0 \%)$ & $0(0 \%)$ & $0(0 \%)$ & NA & $0(0 \%)$ & $0(0 \%)$ & $0(0 \%)$ & NA \\
\hline Bowel & $0(0 \%)$ & $0(0 \%)$ & $0(0 \%)$ & NA & $0(0 \%)$ & $0(0 \%)$ & $0(0 \%)$ & NA \\
\hline Medical & $1(0.1 \%)$ & $1(0.2 \%)$ & $0(0 \%)$ & $1.000^{*}$ & $0(0 \%)$ & $0(0 \%)$ & $0(0 \%)$ & NA \\
\hline Postop complication & $268(29.8 \%)$ & $145(26.0 \%)$ & $123(36.1 \%)$ & 0.001 & $73(36.0 \%)$ & $39(38.2 \%)$ & $11(30.6 \%)$ & 0.711 \\
\hline lleus & $80(8.9 \%)$ & $40(7.2 \%)$ & $40(11.7 \%)$ & 0.020 & $25(12.3 \%)$ & $14(13.7 \%)$ & $1(2.8 \%)$ & $0.189^{*}$ \\
\hline Wound & $58(6.5 \%)$ & $19(3.4 \%)$ & $39(11.4 \%)$ & $<0.001$ & $24(11.8 \%)$ & $12(11.8 \%)$ & $3(8.3 \%)$ & $0.935^{*}$ \\
\hline Dehiscence & $16(1.8 \%)$ & $3(0.5 \%)$ & $13(3.8 \%)$ & $<0.001$ & $10(4.9 \%)$ & $3(2.9 \%)$ & $0(0 \%)$ & $0.446^{*}$ \\
\hline Hernia & $29(3.2 \%)$ & $11(2.0 \%)$ & $18(5.3 \%)$ & 0.007 & $9(4.4 \%)$ & $8(7.8 \%)$ & $1(2.8 \%)$ & $0.444^{*}$ \\
\hline Infection & $17(1.9 \%)$ & $6(1.1 \%)$ & $11(3.2 \%)$ & 0.022 & $8(3.9 \%)$ & $1(1.0 \%)$ & $2(5.6 \%)$ & $0.252^{*}$ \\
\hline Neurological & $3(0.3 \%)$ & $3(0.5 \%)$ & $0(0 \%)$ & $0.293^{*}$ & $0(0 \%)$ & $0(0 \%)$ & $0(0 \%)$ & NA \\
\hline Hematoma & $10(1.1 \%)$ & $6(1.1 \%)$ & $4(1.2 \%)$ & $1.000^{*}$ & $2(1.0 \%)$ & $0(0 \%)$ & $2(5.6 \%)$ & $0.052^{*}$ \\
\hline Medical & $168(18.7 \%)$ & $97(17.4 \%)$ & $71(20.8 \%)$ & 0.204 & $44(21.7 \%)$ & $20(19.6 \%)$ & $7(19.4 \%)$ & 0.895 \\
\hline Pulmonary & $51(5.7 \%)$ & $32(5.7 \%)$ & $19(5.6 \%)$ & 0.913 & $10(4.9 \%)$ & $9(8.8 \%)$ & $0(0 \%)$ & $0.133^{*}$ \\
\hline Cardiac & $41(4.6 \%)$ & $27(4.8 \%)$ & $14(4.1 \%)$ & 0.605 & $10(4.9 \%)$ & $3(2.9 \%)$ & $1(2.8 \%)$ & $0.778^{*}$ \\
\hline Renal & $16(1.8 \%)$ & $8(1.4 \%)$ & $8(2.3 \%)$ & 0.317 & $5(2.5 \%)$ & $2(2.0 \%)$ & $1(2.8 \%)$ & $1.000^{*}$ \\
\hline Urologic & $42(4.7 \%)$ & $26(4.7 \%)$ & $16(4.7 \%)$ & 0.987 & $13(6.4 \%)$ & $2(2.0 \%)$ & $1(2.8 \%)$ & $0.212^{*}$ \\
\hline UTI & $31(3.5 \%)$ & $14(2.5 \%)$ & $17(5.0 \%)$ & 0.049 & $9(4.4 \%)$ & $4(3.9 \%)$ & $4(11.1 \%)$ & $0.218^{*}$ \\
\hline DVT/PE & $19(2.1 \%)$ & $9(1.6 \%)$ & $10(2.9 \%)$ & 0.183 & $3(1.5 \%)$ & $4(3.9 \%)$ & $3(8.3 \%)$ & $0.054^{*}$ \\
\hline
\end{tabular}

Values are presented as the number (\%) of patients.

* Fisher's exact test.

not able to calculate its effect in this cohort, it is an important consideration in future studies.

ALIF-related wound complications in our cohort occurred in $6.5 \%$ of all patients, with a higher rate among obese patients $(11.4 \%$ vs $3.4 \%, p<0.001)$. This was driven primarily by dehiscence $(3.8 \%$ vs $0.5 \%, \mathrm{p}<0.001)$, hernia $(5.3 \%$ vs $2.0 \%, p=0.007)$, and wound infections $(3.2 \%$ vs $1.1 \%, \mathrm{p}=0.022$ ). Obesity has been previously shown to be associated with increased wound complications, including postoperative infection, dehiscence, seroma, and hematoma formation in patients undergoing elective spine surgery. ${ }^{20,21}$ Obesity is also a well-known risk factor for the development of hernias and infections. ${ }^{1,22}$ The increased rate of wound complications in our cohort can be partially explained by increased mechanical stress placed on the anterior incision. Diabetes was also more common among our obese patients $(17.9 \%$ vs $5.7 \%, \mathrm{p}<0.001)$ and is associated with increased risk of impaired wound healing and infection. ${ }^{23}$

Among postoperative medical complications, UTI was the only category that was associated with an increased risk among obese patients $(5.0 \%$ vs $2.5 \%, \mathrm{p}=0.049)$. Previous studies have reported an association between obesity and UTIs. ${ }^{24,25}$ Diabetes is also a well-recognized risk factor for UTI and the proportion of diabetic patients was significantly higher among the obese patients in our cohort. ${ }^{26}$ Another important consideration is urinary catheter duration, which we were unable to determine in this study given its retrospective nature.

Vascular injury is a frequently cited risk of anterior spine exposure, with reported rates ranging from $1.9 \%$ to $6.1 \% .^{27-29}$ Intraoperative vascular injuries in our series occurred in $3.8 \%$ of cases, with venous injuries being more common (3.6\%). Rates were similar among obese and nonobese patients $(3.2 \%$ vs $3.8 \%, \mathrm{p}=0.669)$. Fantini et al. identified osteomyelitis/discitis and previous anterior

\section{TABLE 6. Multivariate analysis for variables associated with postoperative complications in patients who underwent transabdominal retroperitoneal ALIF}

\begin{tabular}{lcc}
\hline \multicolumn{1}{c}{ Variable } & OR $(95 \% \mathrm{Cl})$ & $\mathrm{p} \mathrm{Value}$ \\
\hline Age & $1.026(1.010-1.043)$ & 0.001 \\
\hline Obesity & $1.672(1.177-2.376)$ & 0.004 \\
\hline No. of levels fused & $1.319(1.046-1.663)$ & 0.019 \\
\hline $\begin{array}{l}\text { Degenerative disease or } \\
\text { spondylolisthesis }\end{array}$ & $0.625(0.430-0.907)$ & 0.004 \\
\hline
\end{tabular}




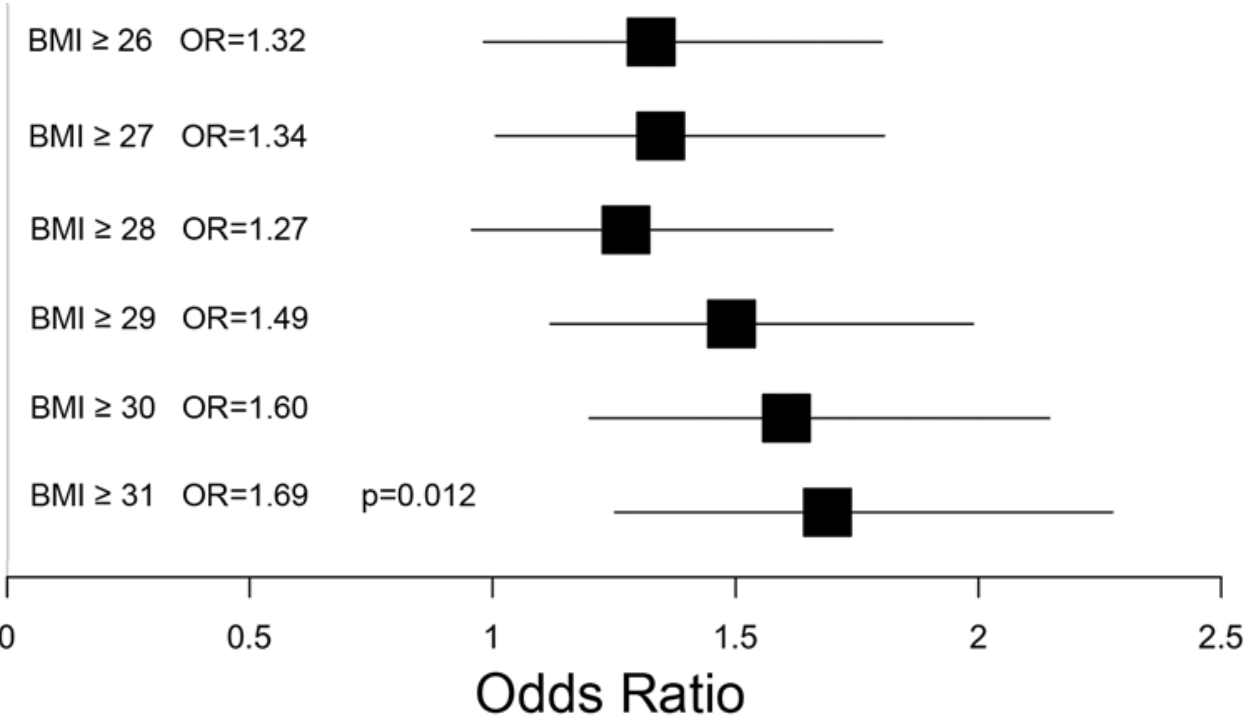

FIG. 1. Identification of a BMI threshold for increased risk of postoperative complication. An estimation table was generated to include candidate cut-points, odds ratios, and corresponding unadjusted and adjusted $p$ values using Bonferroni correction. A BMI threshold of $31 \mathrm{~kg} / \mathrm{m}^{2}$ had the lowest unadjusted $p$ value $(p<0.001)$ and adjusted $p$ value $(p=0.012)$.

spine surgery, but not obesity, as factors associated with an increased risk of vascular surgery in ALIF surgery. ${ }^{28}$ All of our retroperitoneal exposures were performed by experienced vascular surgeons, which contributes to a low rate of vascular injury. In a subgroup analysis of our cohort, we found that osteomyelitis/discitis, noted in 43 patients $(4.6 \%)$, was associated with a nearly fourfold increase in intraoperative venous injury ( $17.1 \%$ vs $4.1 \%$, $\mathrm{p}<0.001)$, but no difference in other postoperative complications. Additionally, rates of osteomyelitis/discitis were not significantly different between obese and nonobese patients in our cohort $(5.1 \%$ vs $4.3 \%, \mathrm{p}=0.568)$.

Another risk of the anterior approach is related to urological complications. The cohort had a rate of $4.7 \%$, and there was no difference between obese and nonobese ( $4.7 \%$ vs $4.7 \%, \mathrm{p}=0.987)$. The most commonly reported complications were urinary retention or incontinence related to medication side effects, not neurological injury, and scrotal edema. Several retrospective analyses and systematic reviews have reported a wide range of rates of retrograde ejaculation in patients who underwent anterior spine surgery. ${ }^{30-33}$ There were no identifiable cases of retrograde ejaculation in our series, but we suspect that this is an underestimate of the true rate given the retrospective nature of this study. We did not identify any cases of intraoperative ureteral injury in patients undergoing transabdominal retroperitoneal ALIF, which is related to two important factors. First, all exposures were performed by experienced vascular surgeons. Second, in high-risk cases, ureteral stents were placed preoperatively to allow for identification of the ureter intraoperatively.

In the 898 patients who underwent transabdominal retroperitoneal ALIF, we identified an increased risk of postoperative complications among obese patients. These differences were driven primarily by higher rates of ileus, wound complications, and UTI. This study is limited by its retrospective design and focuses specifically on perioperative complications rather than long-term complications such as pseudarthrosis, proximal junctional failure, and others. These data suggest a role for careful medical optimization of obese patients prior to transabdominal retroperitoneal ALIF and special attention to their recovery during the postoperative period, with a particular focus on meticulous wound closure, normalizing bowel function, and decreasing urinary catheter duration. It is important to note that despite an increased perioperative risk among obese patients undergoing transabdominal retroperitoneal ALIF, the long-term benefits provided by this approach likely outweigh the short-term risk. Improved restoration of disc height and segmental lordosis ${ }^{17,34}$ may decrease rates of long-term complications and the need for revision surgery, thus justifying this temporary increase in complication profile. Future studies are warranted to determine the long-term benefits of ALIF in obese patients.

\section{Conclusions}

Obesity is associated with an increased rate of postoperative complications in patients undergoing ALIF. This is driven primarily by postoperative, rather than intraoperative, events. Obese patients are at an increased risk of ileus, wound complications, and UTIs compared to nonobese patients. Patients with a BMI $\geq 31 \mathrm{~kg} / \mathrm{m}^{2}$ should be counseled on their increased risk and warrant careful preoperative medical optimization and close monitoring in the postoperative period.

\section{References}

1. Jackson KL II, Devine JG. The effects of obesity on spine surgery: a systematic review of the literature. Global Spine J. 2016;6(4):394-400.

2. Mobbs RJ, Phan K, Malham G, et al. Lumbar interbody fu- 
sion: techniques, indications and comparison of interbody fusion options including PLIF, TLIF, MI-TLIF, OLIF/ATP, LLIF and ALIF. J Spine Surg. 2015;1(1):2-18.

3. Buerba RA, Fu MC, Gruskay JA, et al. Obese Class III patients at significantly greater risk of multiple complications after lumbar surgery: an analysis of 10,387 patients in the ACS NSQIP database. Spine J. 2014;14(9):2008-2018.

4. Burks CA, Werner BC, Yang S, Shimer AL. Obesity is associated with an increased rate of incidental durotomy in lumbar spine surgery. Spine (Phila Pa 1976). 2015;40(7):500-504.

5. Kalanithi PA, Arrigo R, Boakye M. Morbid obesity increases cost and complication rates in spinal arthrodesis. Spine (Phila Pa 1976). 2012;37(11):982-988.

6. Marquez-Lara A, Nandyala SV, Sankaranarayanan S, et al. Body mass index as a predictor of complications and mortality after lumbar spine surgery. Spine (Phila Pa 1976). 2014;39(10):798-804.

7. Patel N, Bagan B, Vadera S, et al. Obesity and spine surgery: relation to perioperative complications. J Neurosurg Spine. 2007;6(4):291-297.

8. Cao J, Kong L, Meng F, et al. Impact of obesity on lumbar spinal surgery outcomes. J Clin Neurosci. 2016;28:1-6.

9. Onyekwelu I, Glassman SD, Asher AL, et al. Impact of obesity on complications and outcomes: a comparison of fusion and nonfusion lumbar spine surgery. J Neurosurg Spine. 2017;26(2):158-162.

10. Sing DC, Yue JK, Metz LN, et al. Obesity is an independent risk factor of early complications after revision spine surgery. Spine (Phila Pa 1976). 2016;41(10):E632-E640.

11. Soroceanu A, Burton DC, Diebo BG, et al. Impact of obesity on complications, infection, and patient-reported outcomes in adult spinal deformity surgery. J Neurosurg Spine. 2015;23(5):656-664.

12. Lucas F, Emery E, Dudoit T, Berger L. Influence of obesity on access-related complications during anterior lumbar spine interbody fusion. World Neurosurg. 2016;92:229-233.

13. Peng CW, Bendo JA, Goldstein JA, Nalbandian MM. Perioperative outcomes of anterior lumbar surgery in obese versus non-obese patients. Spine J. 2009;9(9):715-720.

14. Phan K, Rogers P, Rao PJ, Mobbs RJ. Influence of obesity on complications, clinical outcome, and subsidence after anterior lumbar interbody fusion (ALIF): prospective observational study. World Neurosurg. 2017;107:334-341.

15. Gumbs AA, Bloom ND, Bitan FD, Hanan SH. Open anterior approaches for lumbar spine procedures. Am J Surg. 2007;194(1):98-102.

16. Hsieh PC, Koski TR, O'Shaughnessy BA, et al. Anterior lumbar interbody fusion in comparison with transforaminal lumbar interbody fusion: implications for the restoration of foraminal height, local disc angle, lumbar lordosis, and sagittal balance. J Neurosurg Spine. 2007;7(4):379-386.

17. Phan K, Thayaparan GK, Mobbs RJ. Anterior lumbar interbody fusion versus transforaminal lumbar interbody fusion-systematic review and meta-analysis. Br J Neurosurg. 2015;29(5):705-711.

18. Watkins RG IV, Hanna R, Chang D, Watkins RG III. Sagittal alignment after lumbar interbody fusion: comparing anterior, lateral, and transforaminal approaches. J Spinal Disord Tech. 2014;27(5):253-256.

19. Durand WM, Ruddell JH, Eltorai AEM, et al. Ileus following adult spinal deformity surgery. World Neurosurg. 2018;116:e806-e813.

20. Epstein NE. More risks and complications for elective spine surgery in morbidly obese patients. Surg Neurol Int. 2017;8:66.

21. Piper KF, Tomlinson SB, Santangelo G, et al. Risk factors for wound complications following spine surgery. Surg Neurol Int. 2017;8:269.

22. Bosanquet DC, Ansell J, Abdelrahman T, et al. Systematic review and meta-regression of factors affecting midline incisional hernia rates: analysis of 14,618 patients. PLoS One. 2015;10(9):e0138745.

23. Martin ET, Kaye KS, Knott C, et al. Diabetes and risk of surgical site infection: a systematic review and meta-analysis. Infect Control Hosp Epidemiol. 2016;37(1):88-99.

24. Saliba W, Barnett-Griness O, Rennert G. The association between obesity and urinary tract infection. Eur J Intern Med. 2013;24(2):127-131.

25. Semins MJ, Shore AD, Makary MA, et al. The impact of obesity on urinary tract infection risk. Urology. 2012;79(2):266-269.

26. Nichols GA, Brodovicz KG, Kimes TM, et al. Prevalence and incidence of urinary tract and genital infections among patients with and without type 2 diabetes. J Diabetes Complications. 2017;31(11):1587-1591.

27. Brau SA, Delamarter RB, Schiffman ML, et al. Vascular injury during anterior lumbar surgery. Spine J. 2004;4(4):409412.

28. Fantini GA, Pappou IP, Girardi FP, et al. Major vascular injury during anterior lumbar spinal surgery: incidence, risk factors, and management. Spine (Phila Pa 1976). 2007;32(24):2751-2758.

29. Garg J, Woo K, Hirsch J, et al. Vascular complications of exposure for anterior lumbar interbody fusion. J Vasc Surg. 2010;51(4):946-950.

30. Amaral R, Ferreira R, Marchi L, et al. Stand-alone anterior lumbar interbody fusion-complications and perioperative results. Rev Bras Ortop. 2017;52(5):569-574.

31. Comer GC, Smith MW, Hurwitz EL, et al. Retrograde ejaculation after anterior lumbar interbody fusion with and without bone morphogenetic protein-2 augmentation: a 10-year cohort controlled study. Spine J. 2012;12(10):881-890.

32. Hindoyan K, Tilan J, Buser Z, et al. A retrospective analysis of complications associated with bone morphogenetic protein 2 in anterior lumbar interbody fusion. Global Spine J. 2017;7(2):148-153.

33. Phan K, Lackey A, Chang N, et al. Anterior lumbar interbody fusion (ALIF) as an option for recurrent disc herniations: a systematic review and meta-analysis. J Spine Surg. 2017;3(4):587-595.

34. Dorward IG, Lenke LG, Bridwell KH, et al. Transforaminal versus anterior lumbar interbody fusion in long deformity constructs: a matched cohort analysis. Spine (Phila Pa 1976). 2013;38(12):E755-E762.

\section{Disclosures}

Dr. Tay reports being a consultant for Biomet, Stryker, DePuy Synthes, and Lumetra, and receiving institutional fellowship support from NuVasive and Omega. Dr. Burch reports being a consultant for Medtronic and an ownership stake in Zimmer Biomet. Dr. Berven reports an ownership stake in Green Sun Medical, receiving royalties from Stryker Spine, and being a consultant for Medtronic, Medicrea, and Integrity Spine. Dr. Deviren reports being a consultant for NuVasive, Biomet, Medicrea, Alphatec, and Seaspine; receiving royalties from NuVasive; and receiving institutional fellowship support from Omega and NuVasive. Dr. Dhall reports receiving honoraria from DePuy and Globus. Dr. Chou reports being a consultant for Globus and Medtronic and receiving royalties from Globus. Dr. Mummaneni reports being a consultant for DePuy Synthes, Stryker, and Globus; direct stock ownership in Spinicity/ISD; receiving royalties from DePuy Synthes, Thieme, and Springer; and support from NREF and AOSpine for clinical or research efforts not related to this work. Dr. Ames reports receiving royalties from Stryker, Biomet Zimmer Spine, DePuy Synthes, NuVasive, Next Orthosurgical, K2M, and Medicrea; being a consultant for DePuy Synthes, Medtronic, Stryker, Medicrea, K2M, and Biomet Zimmer; 
research support from Titan Spine, DePuy Synthes, and ISSG; receiving grant funding from SRS; and serving on the editorial board of Operative Neurosurgery, on the executive committee of ISSG, and as a director for Global Spinal Analytics. Dr. Clark reports being a consultant for NuVasive.

\section{Author Contributions}

Conception and design: Clark, Safaee, Tenorio, Choy. Acquisition of data: Clark, Safaee, Tenorio, Osorio, Choy, Amara, Lai. Analysis and interpretation of data: Clark, Safaee, Tenorio, Choy, Molinaro. Drafting the article: Clark, Safaee, Tenorio, Choy. Critically revising the article: Clark, Safaee, Tenorio, Osorio, Choy, $\mathrm{Hu}$, Tay, Burch, Berven, Deviren, Dhall, Chou, Mummaneni,

Eichler, Ames. Reviewed submitted version of manuscript: Clark, Safaee, Tenorio, Osorio. Approved the final version of the manuscript on behalf of all authors: Clark. Statistical analysis: Clark, Safaee, Tenorio, Molinaro, Zhang. Administrative/technical/material support: Clark, Safaee, Tenorio. Study supervision: Clark, Safaee, Tenorio.

\section{Supplemental Information}

Previous Presentations

Portions of this work were presented in abstract form at the 2019

Annual Meeting of the Congress of Neurological Surgeons, San Francisco, California, October 22, 2019.

\section{Correspondence}

Aaron J. Clark: University of California, San Francisco, CA. aaron.clark@ucsf.edu. 